\title{
Experimental study of a fiber Bragg grating accelerometer for seismic measurement
}

\author{
Abraham Pérez-Alonzo' 1 , G. E. Sandoval-Romero² \\ Instituto de Ciencias Aplicadas y Tecnología, Universidad Nacional Autónoma de México, \\ Coyoacán, 04510, Ciudad de México, México \\ ${ }^{1}$ Corresponding author \\ E-mail: ${ }^{1}$ a.perez.iee.daia@gmail.com, ${ }^{2}$ eduardo.sandoval@ccadet.unam.mx
}

Received 13 November 2018; accepted 6 December 2018 DOI https://doi.org/10.21595/vp.2018.20375

Check for updates

Copyright (C) 2019 Abraham Pérez Alonzo, et al. This is an open access article distributed under the Creative Commons Attribution License, which permits unrestricted use, distribution, and reproduction in any medium, provided the original work is properly cited.

\begin{abstract}
This paper presents a fiber Bragg grating accelerometer prototype for seismic applications, including accelerometer scheme, calibration method and accelerometer resonance frequencies. The acceleration calibration and measurements were obtained from the laser beam deflection method. A series of experiments were carried out and results shows an accelerometer sensitivity of $8.07 \mathrm{mV} / \mathrm{g}$ (where $1 \mathrm{~g}=9.81 \mathrm{~m} / \mathrm{s}^{2}$ ) at $0.5 \mathrm{~Hz}$ and $2.63 \mathrm{mV} / \mathrm{g}$ at $50 \mathrm{~Hz}$, the measured range scale goes from 0 to $35 \mathrm{~m} \mathrm{~s}^{-2}$.
\end{abstract}

Keywords: fiber Bragg grating, accelerometer, laser beam deflection.

\section{Introduction}

Due to the great damage caused by the last seismic event in the highly populated Mexico City (September 19th, 2017), it is very important to have an accurate monitoring system in order to reduce casualties and building damages. A monitoring system makes easier to predict the earthquakes, design new buildings models to watch the response against these kinds of events and improve them [1].

Nowadays, there is a wide variety of accelerometers, such as: mechanical, micromechanical, piezoelectric, piezo-resistive, capacitive, heated gas, Hall effect, fiber Bragg grating (FBG) based, among others.

The earthquake monitoring can be performed, among all the accelerometer kinds, by the fiber Bragg grating based accelerometers; FBG sensors have been developed during the last 30 years and have many applications. These kinds of sensors have the next advantages over others: Electro Magnetic Interference (EMI) immunity, Radio Frequency Interference (RFI) immunity, low cost, compact size, multiplexing capacity, and a higher sensitivity (mainly at frequencies under $1 \mathrm{~Hz}$ ) [2-3].

In this work, it is discussed the manufactory and testing of an accelerometer based on a fiber Bragg grating. The results show that this FBG accelerometer provides a ratio of $8.07 \mathrm{mV} / \mathrm{g}$ at $0.5 \mathrm{~Hz}$ and $2.63 \mathrm{mV} / \mathrm{g}$ at $50 \mathrm{~Hz}$ without signal amplification, and an effective frequency bandwidth from 0.01 to $50 \mathrm{~Hz}$ (earthquakes common frequencies range).

\section{Structure and sensing principle of the accelerometer}

\subsection{FBG accelerometer}

Fig. 1 shows the device structure, where a clamped fiber Bragg grating is fixed between a pole and a flexible metal plate. The flexible metal plate acts as an inertial mass. Next to the flexible metal plate there is an electromagnet, where the electromagnet is used to axial strain the FBG sensor by induce an attraction force to the metal plate. 


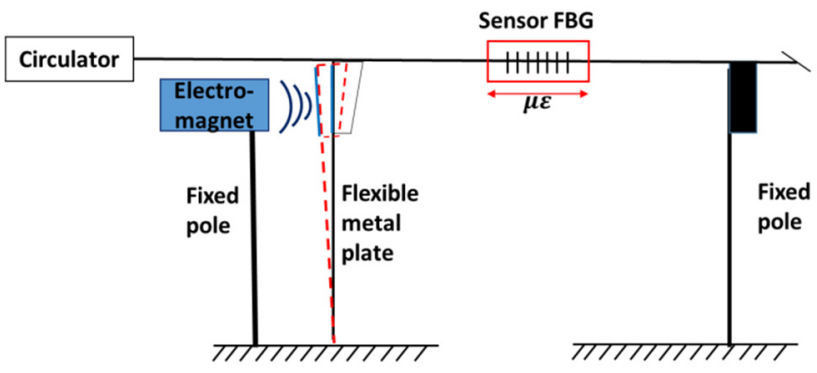

Fig. 1. Accelerometer schematic structure

\subsection{Interrogation system}

For practical field applications, it isn't feasible to use an optical spectrum analyzer due to the big size and weight, as well as the high price. In this work to demodulate the FBG signal, we employ the radiometric interrogation principle, which converts the wavelength shift into an intensity signal. We placed a reference Bragg grating into the optical scheme, shown in Fig. 4, before the sensor FBG. This reference FBG has a spectral overlapping with the sensor FBG. When the sensor is accelerated periodically, the sensor FBG is stretched and after contracted, so the distance between both FBG's Bragg wavelengths is changed.

When the two FBGs have high reflectivity: The incoming light is seen by the first Bragg grating and reflected just at its Bragg wavelength. Therefore, the second Bragg grating does not see all the spectrum of the light source and reflects less intensity in the overlapping region. The higher is the overlap in the Bragg wavelength, the less intensity is reflected by the sensor, the striped area in Fig. 2(a). The sensor therefore operates as an intensity modulating device $[4,5]$.

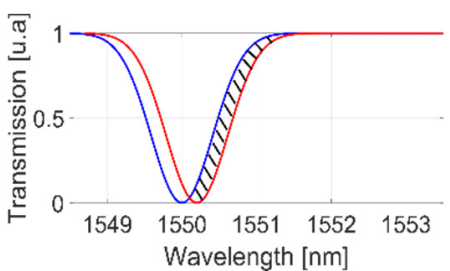

a) Minor reflected power

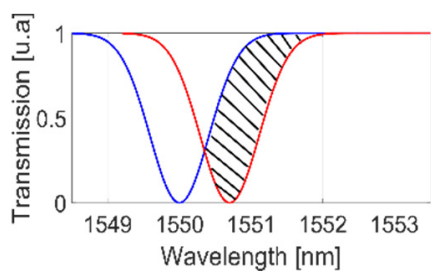

b) Medium reflected power

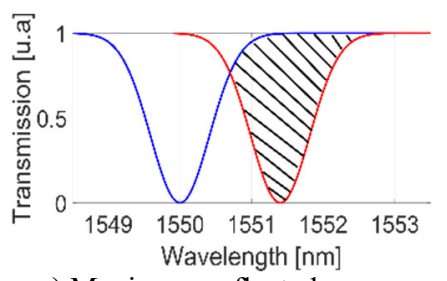

c) Maximum reflected power

Fig. 2. Principle of work of the two overlapping Bragg grating acceleration sensor

\subsection{Laser beam deflection method}

In this experiment, we used the laser beam deflection method as a way to know the acceleration applied to the sensor due to the magnetometer.

This method is an easy way to measure and monitor small displacements $[6,7]$, the scheme used in this experiment is shown in Fig. 3.

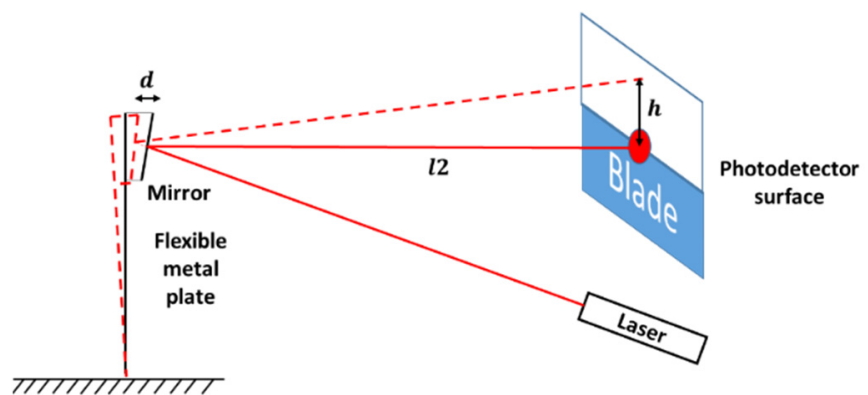

Fig. 3. The laser beam deflection method scheme used in this work 


\section{Experiment}

A series of tests were carried out to verify the accelerometer characteristics, the experimental setup used is shown in Fig. 4 and we employ the beam deflection method to calibrate the device. In this experiment, a small electromagnet generates a series of sine excitations with variable frequency and intensity, which acts as the input signal to the accelerometer, and fiber Bragg grating sensor output voltage is monitored by an oscilloscope.

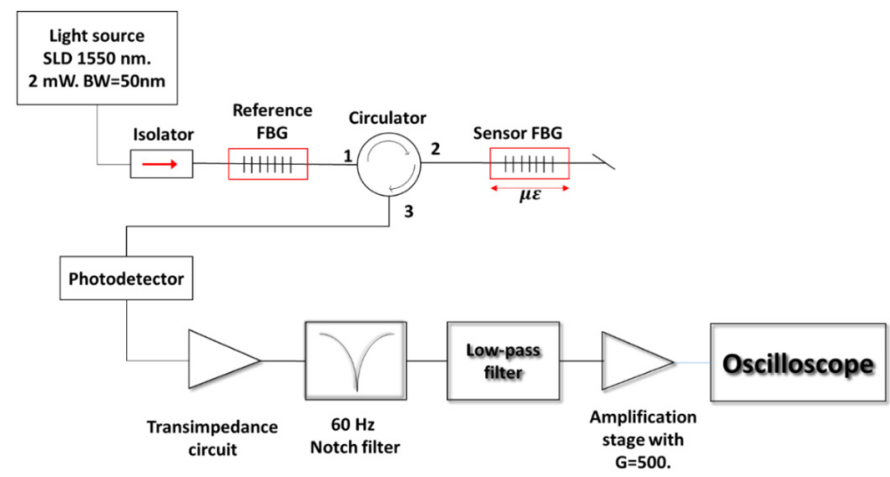

Fig. 4. Experimental setup of the accelerometer

\subsection{Sensitivity}

In order to find the sensor sensitivity, the applied acceleration was varied from $0.08 \mathrm{~g}$ to $3.6 \mathrm{~g}$ $\left(0.78-35.3 \mathrm{~m} / \mathrm{s}^{2}\right)$ at constant frequencies of $0.5,1,5,10,20,30,40$ and $50 \mathrm{~Hz}$, respectively. The sensor output voltage was recorded by the oscilloscope. Fig. 5 shows a linear response between the sensor output voltage and acceleration amplitude applied from 0.5 and $50 \mathrm{~Hz}$, with a sensitivity coefficient of $8.07 \mathrm{mV} / \mathrm{g}(0.5 \mathrm{~Hz})$ and $2.63 \mathrm{mV} / \mathrm{g}(50 \mathrm{~Hz})$, respectively.

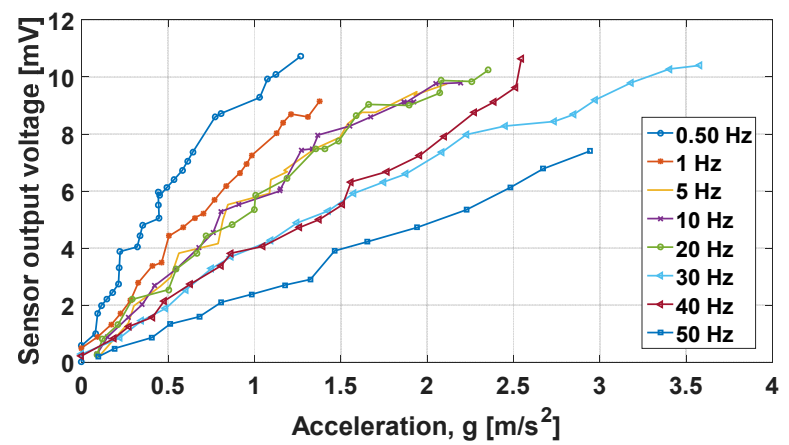

Fig. 5. FBG accelerometer sensitivity at different fixed frequencies excitation

\subsection{Frequency response}

This sensor is meant for seismic application, it must be able to measure low frequencies. To assess the frequency response sensor, the input signal frequency was increased from 0.5 to $300 \mathrm{~Hz}$ with the same exciting amplitude of voltage applied to the electromagnet of $1,2,3$ and $4 \mathrm{~V}$. We used an oscilloscope to obtain the accelerometer output of each frequency and detect the maximum FBG output voltage correspondingly. The amplitude-frequency characteristic of the accelerometer is shown in Fig. 6. The blue line shows an almost flat response range from 5 to $50 \mathrm{~Hz}$ (with a resonant frequency about $74 \mathrm{~Hz}$ ) for $4 \mathrm{Vpp}$ electromagnet excitation voltage, at frequencies below $5 \mathrm{~Hz}$ the sensibility is greater. 


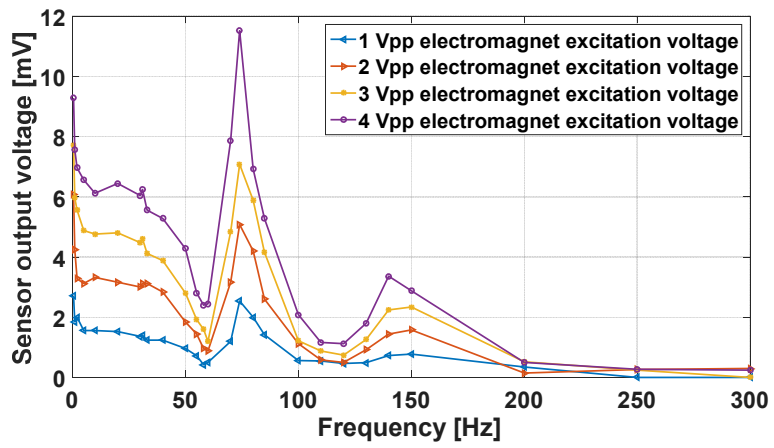

Fig. 6. The frequency response of the accelerometer obtained experimentally at 1,2,3 and $4 \mathrm{Vpp}$ electromagnet excitation voltage

\section{Conclusions}

The acceleration sensor presented in this work, it is sensitive to frequency measurements under $50 \mathrm{~Hz}$. The experimental test and calibration with the laser beam deflection method, shows that the prototype have a good linearity and a wide frequency response range from 0.01 to $50 \mathrm{~Hz}$, a sensitivity coefficient of $8.07 \mathrm{mV} / \mathrm{g}$ at $0.5 \mathrm{~Hz}$ and $2.63 \mathrm{mV} / \mathrm{g}$ at $50 \mathrm{~Hz}$.

The earthquakes common frequencies are below $50 \mathrm{~Hz}$, the earthquake occurred in Mexico City in 2017 had a frequency of $3 \mathrm{~Hz}$, this sensor prototype can measure a range frequency from $0.01 \mathrm{~Hz}$, and so it can be suitable to measure this kind of events.

With this simple interrogation detection scheme employed, the sensor can be made at low cost and feasible for field applications.

\section{Acknowledgements}

This work was supported by DGAPA-UNAM through financing of project PAPIIT IT101019.

\section{References}

[1] Laudati A., Mennella F., Esposito M., et al. A fiber optic Bragg grating seismic sensor. Proceedings of the SPIE, Vol. 19, Issue 24, 2007, p. 1991-1993.

[2] Zhang W., Huang W., Li L., Liu W., Li F. High resolution FBG sensor and its applications in geophysics. 16th International Conference on Optical Communications and Networks (ICOCN), 2017.

[3] Weng Y., Qiao X., Guo T., et al. A robust and compact fiber Bragg grating vibration sensor for seismic measurement. IEE Sensors Journal, Vol. 12, Issue 4, 2011, p. 800-804.

[4] Willsch M., Kraemmer P., Theune N., et al. Highly sensitive micro-mechanical fiber Bragg grating acceleration sensor combined with a new multiplexable interrogation principle. Proceedings of the SPIE, Vol. 4074, 2000, p. 45-53.

[5] Lobo A., Ferreira, L., Santos J., Jackson D. Analysis of the reflective-matched fiber Bragg grating sensing interrogation scheme. Applied Optics, Vol. 36, Issue 4, 1997, p. 934-939.

[6] Putman C., De Grooth B., Van N., Greve J. A detailed analysis of the optical beam deflection technique for use in atomic force microscopy. Journal of Applied Physics, Vol. 72, Issue 1, 1992, p. 6-12.

[7] Beaulieu L., Godin M., Laroche O., et al. A complete analysis of the laser beam deflection systems used in cantilever-based systems. Ultramicroscopy, Vol. 107, Issues 4-5, 2007, p. 422-430. 